\title{
Correction to: Chronic unpredictable mild stress produces depressive-like behavior, hypercortisolemia, and metabolic dysfunction in adolescent cynomolgus monkeys
}

Teng Teng, Carol A. Shively $\mathbb{D}$, Xuemei Li, Xiaofeng Jiang, Gretchen N. Neigh $\mathbb{D}$, Bangmin Yin, Yuqing Zhang, Li Fan, Yajie Xiang, Mingyang Wang, Xueer Liu, Mengchang Qin, Xinyu Zhou and Peng Xie (1)

Correction to: Translational Psychiatry https://doi.org/10.1038/s41398-020-01132-6 published online 04 January 2021

The original version of this article unfortunately contained a mistake in the Acknowledgement section. The acknowledgement of funding from National Institutes of
Health grant number R01HL087103 was incorrect. No funding from the grant was used to support the study. The authors apologize for the mistake. The original article has been corrected.

Published online: 15 February 2021 